\title{
BMI in der Jugend sagt späteres KHK- und Diabetesrisiko voraus
}

\author{
Welche Bedeutung hat ein - auch \\ nur grenzwertig - erhöhter Body- \\ Mass-Index (BMI) in der Jugend als \\ möglicher Risikofaktor für spätere, \\ mit Übergewicht assoziierte Krank- \\ heiten, einschließlich Diabetes Typ 2 \\ und koronare Herzerkrankung?
}

- Grundlage der Studie waren die Daten des „Metabolic, Lifestyle and Nutrition Assessment in Young Adults (MELANY-Studie) der Israelischen Armee (IDF) an 37000 gesunden jungen Männern, deren BMI in der Jugend und im jungen Erwachsenenalter gemessen worden war. Diese Personen wurden periodisch ab einem Alter von etwa 25 Jahren alle drei bis fünf Jahre weiter medizinisch untersucht. Erfasst wurden demografische und medizinische Daten mithilfe von Fragebögen und Tests. Dann wurden die Daten in einem zentralen Computer gespeichert.

In die Studie wurden primär 37674 gesunde junge Männer mit einem BMI von 15-36 aufgenommen. Wiederholt über 17,4 Jahre erfolgten Messungen von Größe, Gewicht und BMI. Bekannte Diabetiker und Patienten mit KHK waren schon zu Beginn ausgeschlossen. Ebenso wurden 135 neu entdeckte Diabetiker nicht aufgenommen. Während der Studie entwickelten 1173 Männer Diabetes (Nüchtern-BZ $>126 \mathrm{mg} / \mathrm{dl}$ ), davon $20(1,7 \%)$ Typ 2.

Die Beobachtungen wurden beendet, wenn während der Studie Diabetes oder KHK angiografisch ( $>50 \%$-Stenose) nach einem Stress-Test diagnostiziert wurden oder die Männer starben $(\mathrm{n}=127)$. Die Beobachtungszeit war im Mittel 17,4 Jahre, 96,4\% wurden mindestens zwölf Jahre beobachtet.

In einem Alter von 17,4 Jahren lagder BMI zwischen 17,3 bis 27,6 (51,9 bis 83,8 kg). Das mittlere Alter der Erwachsenen war bei der ersten Nachuntersuchung 30,6 Jahre und der BMI lag zwischen 21,4 und 30,6. Systolischer und diastolischer Blutdruck, Puls und nüch-

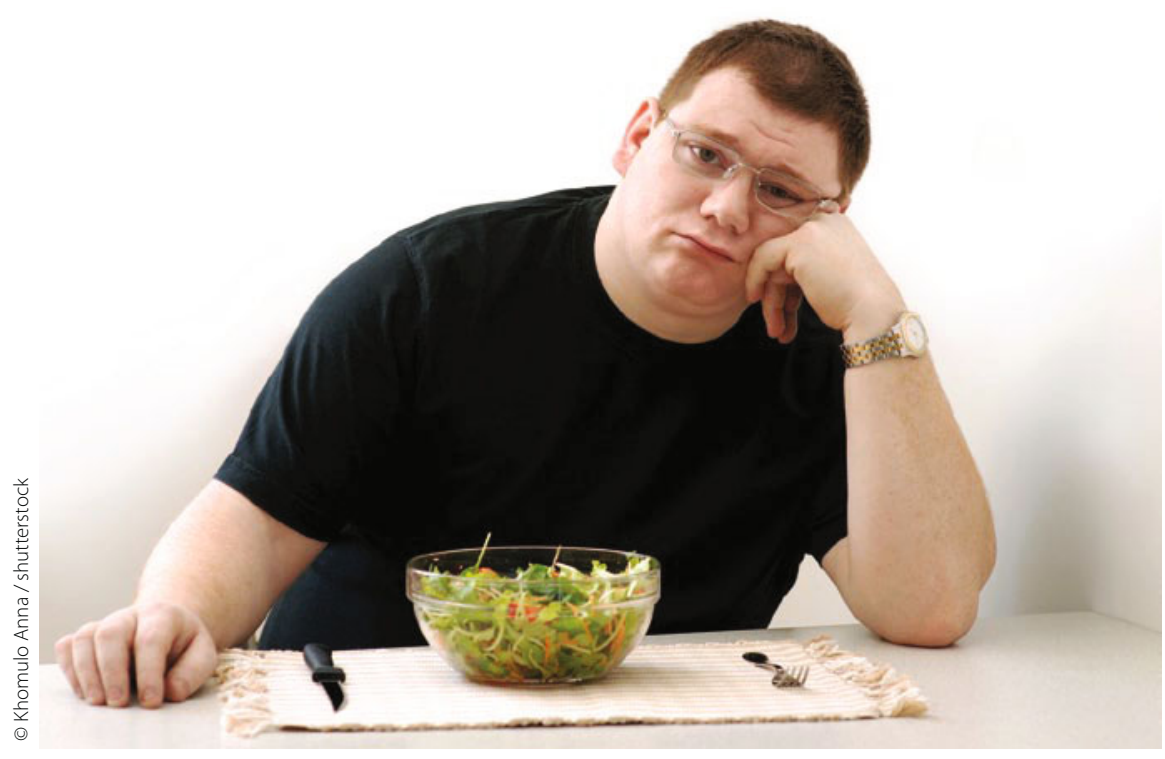

Bereits im frühen Erwachsenenalter wird der Grundstein für KHK und Diabetes gelegt.

tern-BZ, LDL-Cholesterin, Triglyzeride und Leberwerte stiegen progressiv pro Dezile des adoleszenten BMI an, während HDL-Cholesterin abnahm (alle Vergleiche $\mathrm{p}<0,001$ für die Trends).

Während der 650000 Personenjahre unter Beobachtung wurden neben den 1173 Typ-2-Diabetikern auch 327 Fälle mit KHK angiografisch diagnostiziert. In einer multivariaten Analyse war der BMI im Adoleszentenalter ein Prädiktor für die spätere Diagnose Diabetes mellitus mit einem für die drei höchsten BMI-Dezilen gegenüber der untersten BMI-Dezile signifikant erhöhten Risiko. Ebenso war der BMI im Adoleszentenalter ein signifikanter Prädiktor für spätere KHK für den gesamten BMI-Bereich. Das Risiko für KHK stieg für jede BMI-Einheit jeweils um $12 \%$ an.

Der BMI im Alter von 17 Jahren ist also ein unabhängiger Prädiktor für koronare Herzerkrankung im jungen Erwachsenenalter, auch wenn er sich noch im etablierten Normbereich befindet. Obwohl der BMI im Adoleszentenalter auch ein Prädiktor für das Risiko für späteres Auftreten eines Diabetes mellitus ist, ist dieses Risiko nicht unabhän- gig von dem Risiko, das durch einen BMI im Erwachsenenalter vorhergesagt wird.

\section{Kommentar}

Bemerkenswerterweise war in dieser großen Untersuchung bei jungen Männern der Body-Mass-Index ein substanzieller Risikofaktor für Adipositas-assoziierte Krankheiten im mittleren Lebensabschnitt. Obwohl der Diabetes mellitus hauptsächlich mit dem BMI assoziiert ist, der zum Zeitpunkt der Diagnose vorliegt, ist das Risiko für koronare Herzerkrankung sowohl mit einem erhöhten BMI im Adoleszentenalter, als auch im Erwachsenenalter assoziiert.

Die Ergebnisse der Arbeit legen nahe, dass die Prozesse, die der Entwicklung einer koronaren Herzerkrankung zugrunde liegen, vor allem die Arteriosklerose, gradueller und in einem strukturellen Umbau resultierend ablaufen, während die Prozesse, die zu einem Diabetes mellitus führen, zunächst potentiell reversibel und damit weniger graduell stattfinden.

E. FRITSCHKA =

\section{- A. Tirosh et al.}

Adolescent BMI trajectory and risk of diabetes versus coronary disease. N. Engl. J. Med 364 (2011) 1315-1325 